\title{
INTEROBSERVER AGREEMENT OF PI-RADS SCORE VERSION 2 FOR PROSTATE CANCER
}

\author{
Chatwadee Limpaiboon *, Nisachon Chatangam **, Pattarin Burapasomboon **, Kewalee Sasiwimonphan ** \\ *Department of Radiology, Faculty of Medicine, Chulalongkorn University, Department of Radiology, \\ Phramongkutklao Hospital, Bangkok, Thailand \\ **Department of Radiology, King Chulalongkorn Memorial Hospital, The Thai Red Cross Society, \\ Department of Radiology, Faculty of Medicine, Chulalongkorn University, Bangkok, Thailand
}

Objective: To assess the interobserver agreement with the use of the Prostate Imaging Reporting and Data System (PI-RADS), version 2.0 to detect prostate cancer using multiparametric magnetic resonance (MR) imaging in a tissue diagnosis patient population.

Materials and Methods: Fifty-six lesions in 37 patients with elevated prostate-specific antigen levels who underwent transrectal ultrasound (TRUS)-guided biopsy or prostatectomy with multiparametric MR imaging of prostate suspicious lesions detected using multiparametric MR imaging were scored by 4 readers of varying experience who were blinded to pathologic results using the newly revised PI-RADS and the scoring system version 2. Inter-observer agreement was evaluated using the Intra-Interclass Correlation Coefficient (ICC).

Results: Inter-observer agreement of 4 observers using the PI-RADS, v2 for prostate cancer produced an intermediate to good agreement beyond chance; ICC $=0.736,(95 \% \mathrm{CI}: 0.565,0.851)$. Concerning all observers, the most correlated PI-RADS assessment was between observers 1 and $2, \mathrm{r}=0.758(p<0.001)$.

Conclusion: PI-RADS score, version 2 showed intermediate to good agreement in readers of varying experience.

Keywords : Prostate cancer, Prostate MRI, PI-RADS, version 2, Gleason score

J Southeast Asian Med Res 2017; 1: 63-69.

http://www.jseamed.org

\section{Correspondence to:}

Limpaiboon C, Department of Radiology, Phramongkutklao Hospital, Bangkok 10400, Thailand

E-mail : chatmed10@yahoo.com 


\section{Introduction}

Prostate cancer is the second most frequently diagnosed cancer worldwide ( $13.6 \%$ of all diagnosed cancers) and the third most lethal cancer in men in the developed world. ${ }^{(1)}$ The detection rate of prostate cancer is only around $25 \%$ when based on elevated prostate-specific antigen (PSA) and digital rectal examination. Prostate MRI has become an increasingly common adjunctive procedure used to detect prostate cancer. Clinical applications of prostate MRI have expanded to include, not only locoregional staging, but also tumor detection, localization (registration against an anatomical reference), characterization, risk stratification, surveillance, assessment of suspected recurrence and image guidance for biopsy, surgery, focal therapy and radiation therapy.

The procedure of choice is multiparametric MRI (mp MRI), a combination of high-resolution T2-weighted (T2W) morphological sequences and the multiparametric techniques of diffusion-weighted MRI (DWI), dynamic contrast-enhanced MRI (DCE-MRI) and proton MR spectroscopy ( $\left.{ }^{1} \mathrm{H}-\mathrm{MRS}\right)$. Previously, no uniform recommendations existed in the form of guidelines to implement and standardize communication of findings. The European Society of Urogenital Radiology (ESUR) in 2012 established clinical guidelines to acquire, interpret, and report mp MRI of the prostate to facilitate a greater level of standardization and consistency. These recommendations, popularly referred to as Prostate Imaging Reporting and Data System (PI-RADS), ${ }^{(2)}$ were based on literature evidence and consensus expert opinion and were modeled on similar efforts in mammography (BI-RADS), ${ }^{(3)}$ leading to the transformation of breast cancer care. A number of studies have subsequently validated PI-RADS in certain research and clinical settings; however, experience has highlighted some limitations, in part due to technical improvements and also changes in clinical practice ${ }^{(4-18)} \mathrm{A}$ joint steering committee formed by the American College of Radiology (ACR), ESUR and the nonprofit organization AdMeTech Foundation, have recently attempted to update and improve on the original proposals. PI-RADS version 2 (v2) was officially launched at RSNA 2014, and is now available online. ${ }^{(19)}$ Research has shown substantial inter-observer variability in the interpretation of prostate MRI as a result of heterogeneous reader experience.
The purpose of this study was to assess the inter-observer agreement of the PI-RADS score using histology obtained from TRUS biopsy or pathological results from prostatectomy as the reference standard.

\section{Materials and Methods}

Patient cohort

This retrospective study was approved by the Institutional Review Board and required neither patient approval nor informed consent to review patients' images and medical records. However, written informed consent was obtained from all patients for TRUS biopsy or prostatectomy before each procedure according to our hospital's regular policy. A total of 37 patients with increased prostate-specific antigen (PSA) levels (above $4 \mathrm{ng} / \mathrm{ml}$ ) were retrospectively recruited and enrolled in a research database from April 1,2013 December 31, 2014. Inclusion criteria were patients who had biopsy proven prostate cancer and undergone mp MRI without endorectal coil to detectprostate cancer, including high-resolution, triplanar T2-weighted anatomic imaging, diffusion-weighted imaging (DWI), proton MR spectroscopy (1H-MRS) and DCE- MRI at 3 Tesla (3T) in KCMH before surgery. Exclusion criteria included patients who had incomplete pathological data, image distortion caused by hip prostheses or patient motion too severe for readers to interpret and short interval between MRI and biopsy (less than 4 weeks).

\section{MR imaging}

All imaging studies were performed using a thirtytwo-channel cardiac coil parallel imaging (sensitivity-encoding [SENSE]; Philips Medical Systems, Best, the Netherlands) technique with a 3-T magnet (Achieva; Philips Medical Systems) without the use of an endorectal coil and prior bowel preparation. Hyoscine butylbromide (Buscopan; Boehringer Ingelheim, Ingelheim, Germany) (20 mg) was injected intravascularly immediately before beginning the MR imaging examination to reduce peristalsis. T2-weighted turbo spin-echo images were acquired in three orthogonal planes (transverse, sagittal, and coronal). T2WI scan parameters were as follows: repetition time (TR)/ echo time (TE), 2740/58 msec; slice thickness, $3 \mathrm{~mm}$; interslice gap, $0 \mathrm{~mm}$; field of view (FOV), $1.4 \mathrm{~cm}$; number of signals acquired (NSA), 3 and sensitivity encoding (SENSE) factor, 2 . 
Axial T1-weighted turbo field echo sequences (6-mm slice thickness; FOV, $3.8 \mathrm{~cm}$ ) were acquired to detect biopsy artifacts and assess lymph nodes. Diffusion-weighted MR images were acquired in the transverse plane using the single-shot echo planar imaging (EPI) technique with parallel imaging and fat suppression (spectral attenuation inversion recovery). Scan parameters were as follows: TR/TE, 2130-2582/63-76; slice thickness, $3 \mathrm{~mm}$; interslice gap, $0 \mathrm{~mm}$; FOV, $20 \mathrm{~cm}$; SENSE factor 2; and NSA, 3. The frequency direction was anteroposterior to decrease motion or susceptibility artifacts over the prostate. Diffusion-encoding gradients were applied as $4 \mathrm{~b}$ values from $500-1500 \mathrm{~s} / \mathrm{mm}^{2}$ $\left(500,1000,1200\right.$ and $\left.1500 \mathrm{~s} / \mathrm{mm}^{2}\right)$ along the three orthogonal directions of motion-probing gradients. The $\mathrm{b}$ values were varied by changing the amplitude of the diffusion gradient with all timing parameters fixed. The ADC maps were automatically constructed on a pixel-by-pixel basis (for b values of 0 and for each $b$ value from $500-1500 \mathrm{~s} / \mathrm{mm}^{2}$ ). The DWI acquisition time was less than 10 minutes.

\section{Scoring system}

The ESUR guidelines endorse a division of the prostate gland in 39 regions. ${ }^{(19)}$ All lesions are rated on a score from the three MRI sequences (T2WI, DWI, DCE-MRI) according to PI-RADS v2. ${ }^{(19)}$ To evaluate T2-weighted data sets, the location of the lesion either in the peripheral or the central zone has to be considered Table 1-5.

Table 1. PI-RADS v2. Assessment Categories

PI-RADS I Very low (clinically significant cancer is highly unlikely to be present)
PI-RADS 2 Low (clinically significant cancer is unlikely to be present)
PI-RADS 3 Intermediate (the presence of clinically significant cancer is equivocal)
PI-RADS 4 High (clinically significant cancer is likely to be present)
PI-RADS 5 Very high (clinically significant cancer is highly likely to be present)

Table 2. Peripheral zone (PZ) and Transitional zone (TZ) Peripheral zone (PZ)

\begin{tabular}{cccc}
\hline DWI & T2W & DCE & PI-RADS \\
\hline 1 & any* & any & 1 \\
2 & any & any & 2 \\
3 & any & negative/positive & $3 / 4$ \\
4 & any & any & 4 \\
5 & any & any & 5 \\
\hline
\end{tabular}

Transitional zone (TZ)

\begin{tabular}{cccc}
\hline T2W & DWI & DCE & PI-RADS \\
\hline 1 & any & any & 1 \\
2 & any & any & 2 \\
3 & $\leq 4 / 5$ & any /any & $3 / 4$ \\
4 & any & any & 4 \\
5 & any & any & 5 \\
\hline
\end{tabular}

*any indicates 1-5

Table 3. PI-RADS assessment for $\mathrm{T} 2 \mathrm{~W}$ at Peripheral zone (PZ) and Transitional zone (TZ)

\begin{tabular}{|c|c|}
\hline Score & Peripheral zone (PZ) \\
\hline 1 & Uniform hyperintense signal intensity (normal) \\
\hline 2 & $\begin{array}{l}\text { Linear or wedge-shaped hypointensity or diffuse mild hypointensity, } \\
\text { usually indistinct margin }\end{array}$ \\
\hline 3 & $\begin{array}{l}\text { Heterogeneous signal intensity or non-circumscribed, rounded, } \\
\text { moderate hypointensity (Includes others that do not qualify as } 2,4 \text {, } \\
\text { or } 5 \text { ) }\end{array}$ \\
\hline 4 & $\begin{array}{l}\text { Circumscribed, homogenous moderate hypo intensefocus/mass } \\
\text { confined to prostate and }<1.5 \mathrm{~cm} \text { in greatest dimension }\end{array}$ \\
\hline 5 & $\begin{array}{l}\text { Same as } 4 \text { but } \geq 1.5 \mathrm{~cm} \text { in greatest dimension or definite extraprostatic } \\
\text { extension/invasive behavior }\end{array}$ \\
\hline Score & Transition zone (TZ) \\
\hline 1 & Homogeneous intermediate signal intensity (normal) \\
\hline 2 & $\begin{array}{l}\text { Circumscribed hypointense or heterogeneous encapsulated nodule(s) } \\
\text { (BPH) }\end{array}$ \\
\hline 3 & $\begin{array}{l}\text { Heterogeneous signal intensity with obscured margins. Includes others } \\
\text { that do not qualify as } 2,4 \text { or } 5\end{array}$ \\
\hline 4 & $\begin{array}{l}\text { Lenticular or noncircumscribed, homogeneous, moderately } \\
\text { hypo-intense, and }<1.5 \mathrm{~cm} \text { in greatest dimension }\end{array}$ \\
\hline 5 & $\begin{array}{l}\text { Same as } 4 \text {, but } \geq 1.5 \mathrm{~cm} \text { in greatest dimension or definite extraprostatic } \\
\text { extension/invasive behavior }\end{array}$ \\
\hline
\end{tabular}

Table 4. PI-RADS assessment for DWI and ADC

\begin{tabular}{cl}
\hline Score & Peripheral zone (PZ) or Transition zone (TZ) \\
\hline 1 & No abnormality (i.e., normal) on ADC and high b-value DWI \\
2 & Indistinct hypointense on ADC \\
3 & Focal mildly/moderately hypointense on ADC and \\
& iso intense/mildly hyperintense on high b-value DWI \\
4 & Focal markedly hypointense on ADC and markedly \\
& hyperintense on high b-value DWI; < 1.5 cm in greatest \\
& dimension \\
& Same as 4 but $\geq 1.5 \mathrm{~cm}$ in greatest dimension or definite \\
& extraprostatic extension/invasive behavior
\end{tabular}


Table 5. PI-RADS assessment for DCE

\begin{tabular}{cl}
\hline Score & \multicolumn{1}{c}{ Peripheral zone (PZ) or Transition zone (TZ) } \\
\hline negative & $\begin{array}{l}\text { No early enhancement, or diffuse enhancement not corresponding to a } \\
\text { focal finding on T2W and/or DWI or focal enhancement corresponding } \\
\text { to a lesion demonstrating features of BPH on T2WI }\end{array}$ \\
positive $\quad$ Focal, and; earlier than or contemporaneously with enhancement of \\
adjacent normal prostatic tissues, and; corresponds to suspicious finding \\
on T2W and/or DWI
\end{tabular}

\section{Scoring}

Lesions ( $\mathrm{n}=37$ ) were retrospectively evaluated by four blinded readers \{K.S., C.L., N.C. and P.B. with 8 years' (abdominal radiologist), 1 year 6 months' (second year fellowship training in advanced body imaging), 6 month's (first year fellowship training in advanced body imaging) of experience and no-self interpretation of mp MRI of the prostate (third year radiology resident), respectively\} comprising the different MRI sequences (T2WI, DWI, DCE-MRI). Third year radiology resident was required to attend lectures for about 3 months given by an abdominal radiologist with 8 years' of experience in interpreting $\mathrm{mp}$ MRI of the prostate. Scoring was performed according to the ESUR guidelines (PI-RADS v2). All readers evaluated each lesion separately and were blinded with respect to the patients' clinical data and the histology of the corresponding TRUS biopsy or pathological result. All lesions were marked by a circle on the PACS workstation before starting the study evaluation Fig. 1, 2. Lesion documentation used the segmentation model 39 -regions; 36 for the prostate, two for seminal vesicles and one for the external urethral sphincter. ${ }^{(19)}$ Fig. 3
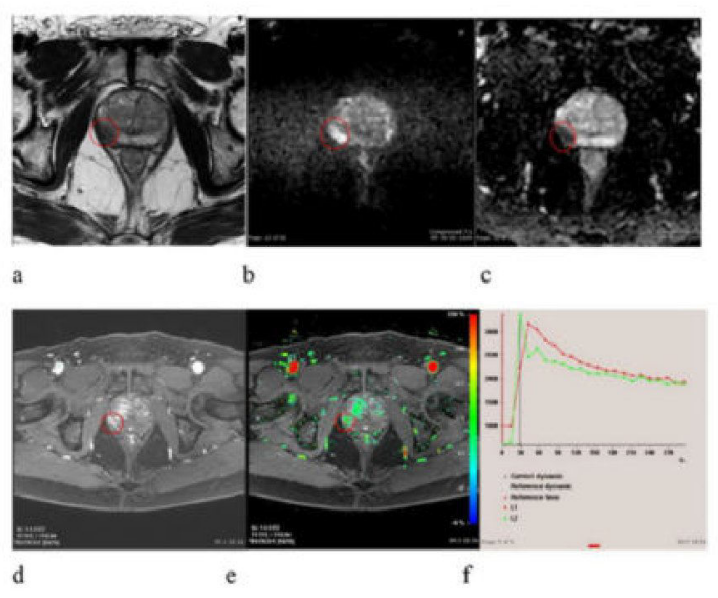

Fig. 1 Example of prostate MRI evaluation of an Axial T2WI with a $1.3 \mathrm{~cm}$ circumscribed lesion located in the right posterolateral aspect of PZ at midgland level (marked with a circle); b, c corresponding apparent diffusion coefficient (ADC) map showing a reduced signal and diffusion-weighted imaging (DWI) on high b value $\left(1,500 \mathrm{~s} / \mathrm{mm}^{2}\right)$; d, e, f related dynamic contrast enhanced (DCE)-MRI with steep initial slope of contrast media uptake followed by a quick washout (type 3 curve).

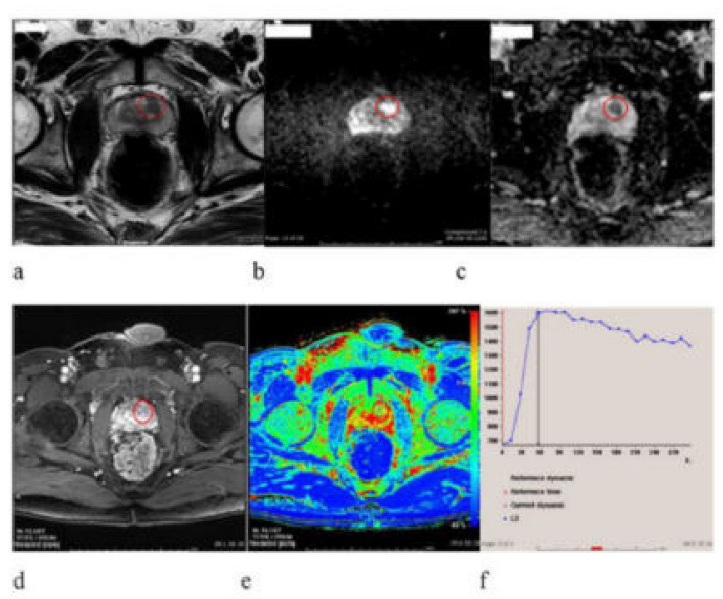

Fig. 2 Example of prostate MRI evaluation of an Axial T2WI with a $0.8-\mathrm{cm}$ circumscribed lesion located in the left anterior aspect of $\mathrm{TZ}$ at midgland level (marked with a circle); $b$, c corresponding apparent diffusion coefficient (ADC) map showing a reduced signal and diffusion-weighted imaging (DWI) on high b value $\left(1,500 \mathrm{~s} / \mathrm{mm}^{2}\right)$; $\mathrm{d}, \mathrm{e}, \mathrm{f}$ related dynamic contrast enhanced (DCE)-MRI with steep initial slope of contrast media uptake followed by a quick washout (type 3 curve).

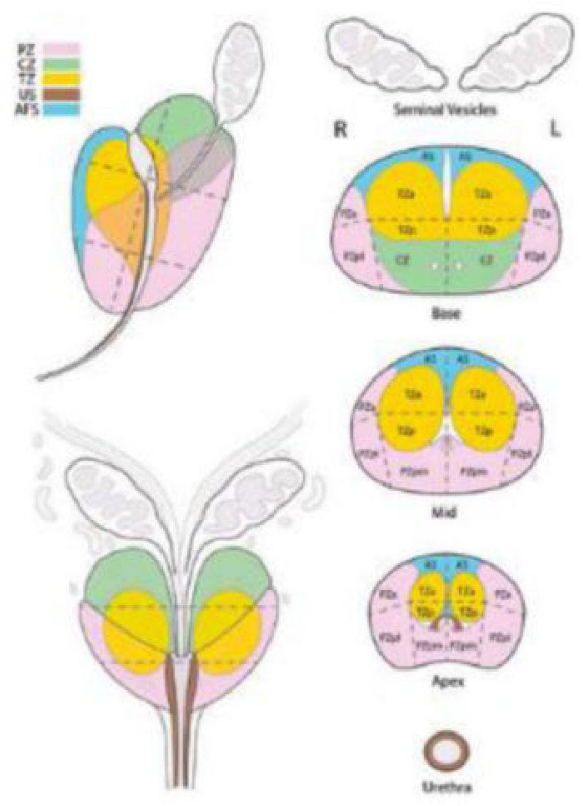

Fig. 3 The segmentation model 39-regions; 36 for the prostate, two for seminal vesicles and one for the external urethral sphincter.

\section{Statistical analysis}

Pearson's correlation test was performed for inter-observer correlation. The Intra-Interclass Correlation Coefficient (ICC) was calculated for 1) inter-observer agreement of PI-RADS 
score for prostate cancer, 2) agreement between location of tumor (TZ only, PZ only, both TZ and PZ) and low grade lesion (Gleason score $<7$ ) with moderate to high grade lesion (Gleason score $\geq 7$ ) and 3) agreement between moderate to high grade lesion (Gleason score $\geq 7$ ) and PI-RADS score. The Chi-square test and ANOVA test were used to determine association between Gleason score $(<7$ and $\geq 7)$ and location of tumor (transitional zone and/or peripheral zone). We performed all calculations with SPSS statistics, 20.0, SPSS (IBM, Corp. Armonk, NY). Statistical significance was defined at a $p$ value below 0.05 .

\section{Results}

\section{Demographic data}

Thirty-seven patients were confirmed histopathologically to have prostate adenocarcinoma. The mean age of all patients was $65.24 \pm 6.62$ years (median 65 years) and mean PSA value was $17.8 \pm 25.33 \mathrm{ng} / \mathrm{ml}($ median $8.25 \mathrm{ng} / \mathrm{ml}$ )

\section{Table 6.}

Table 6. Mean, SD and median of age and PSA of the study population.

\begin{tabular}{lccc}
\hline & Valid N & Mean \pm SD & Median [Min, Max $]$ \\
\hline Age (years) & 37 & $65.24 \pm 6.62$ & $65[51,77]$ \\
PSA (ng/ml) & 28 & $17.8 \pm 25.33$ & $8.25[2,103.7]$ \\
\hline
\end{tabular}

\section{Gleason score and MR imaging}

No significant relationship was found between incidence of location of tumor in TZ only, PZ only, both TZ and PZ (overall 56 lesions) and Gleason score $<7$ and $\geq 7$ ( $p$-value $=0.710)$ Table 7.

Table 7. Incidence of location of tumor in TZ only, PZ only, both $\mathrm{TZ}$ and $\mathrm{PZ}$ (overall 56 lesions) and Gleason score $<7$ and $\geq 7$.

\begin{tabular}{cccc}
\hline Zone & $\begin{array}{c}\text { Gleason }<7 \\
(\mathrm{n}=27)\end{array}$ & $\begin{array}{c}\text { Gleason } \geq 7 \\
(\mathrm{n}=29)\end{array}$ & $p$-value \\
\hline TZ only & $4(14.8 \%)$ & $6(20.7 \%)$ & 0.710 \\
PZ only & $22(81.5 \%)$ & $21(72.4 \%)$ & \\
Both TZ and PZ & $1(3.7 \%)$ & $2(6.9 \%)$ & \\
\hline
\end{tabular}

\section{Inter-observer agreement}

Inter-observer agreement of four observers using the PI-RADS $\mathrm{v} 2$ for prostate cancer was in intermediate to good agreement, ICC = $0.736,(95 \% \mathrm{Cl}: 0.565,0.851)$. The most correlated PI-RADS assessment was found between observers 1 and $2, r=0.758$ $(p<0.001)$ followed by observers 1 and $3, r=0.579(p<0.001)$

Table 8.

Table 8. Correlation and $p$-value of four observers

\begin{tabular}{|c|c|c|c|c|c|c|c|c|}
\hline \multirow{2}{*}{$\begin{array}{c}\text { PI-RADS } \\
\text { score }\end{array}$} & \multicolumn{2}{|c|}{ Observer I } & \multicolumn{2}{|c|}{ Observer 2} & \multicolumn{2}{|c|}{ Observer 3} & \multicolumn{2}{|r|}{ Observer 4} \\
\hline & r & p-value & r & p-value & $r$ & p-value & r & p-value \\
\hline Observer 2 & 0.758 & $<0.001$ & - & - & 0.499 & 0.002 & 0.476 & 0.003 \\
\hline Observer 3 & 0.579 & $<0.001$ & 0.499 & 0.002 & - & - & 0.390 & 0.017 \\
\hline Observer 4 & 0.431 & 0.008 & 0.476 & 0.003 & 0.390 & 0.017 & $=$ & . \\
\hline
\end{tabular}

In the subgroup, excellent agreement was found among four observers, $\mathrm{ICC}=0.972,(95 \% \mathrm{CI}: 0.915,0.995)$ between location of tumor (TZ only, PZ only, both TZ and $\mathrm{PZ}$ ) and low grade to high grade lesion (Gleason score $<7$ and $\geq 7$ ) Table 9.

Table 9. Inter-observer agreement between location of tumor in each zone of prostate gland and Gleason score $<7$ and $\geq 7$ using intra-Interclass Correlation Coefficient $(\mathrm{ICC})=0.972,(95 \% \mathrm{CI}$ : $0.915,0.995$ ).

\begin{tabular}{cccccccc}
\hline Observer & \multicolumn{2}{c}{$\begin{array}{c}\mathrm{TZ} \\
\text { (lesions) }\end{array}$} & $\begin{array}{c}\mathrm{PZ} \\
\text { (lesions) }\end{array}$ & $\begin{array}{c}\mathrm{TZ} \text { and } \mathrm{PZ} \\
\text { (lesions) }\end{array}$ & $\begin{array}{c}\text { benign } \\
\text { (lesions) }\end{array}$ \\
\hline 1 & 2 & 6 & 17 & 21 & 1 & 2 & 7 \\
2 & 2 & 6 & 16 & 21 & 2 & 2 & 7 \\
3 & 1 & 4 & 15 & 23 & 1 & 2 & 10 \\
4 & 2 & 10 & 14 & 21 & 1 & 1 & 7 \\
\hline
\end{tabular}

Excellent agreement was found among four observers, ICC $=0.903$, (95\% CI: $0.575,0.997)$ between moderate to high grade lesion (Gleason score $\geq 7$ ) in TZ only and given PI-RADS score of all observers Table 10.

Table 10. Inter-observer agreement between moderate to high grade lesion (Gleason score $\geq 7$ ) in TZ only and PZ only and PI-RADS score using intra-Interclass Correlation Coefficient (ICC) $=0.903$, $(95 \% \mathrm{CI}: 0.575,0.997)$ and $\mathrm{ICC}=0.542,(95 \% \mathrm{CI}: 0.009,0.982)$ respectively.

\begin{tabular}{cccccccc}
\hline \multirow{2}{*}{$\begin{array}{c}\text { Observer } \\
\end{array}$} & \multicolumn{3}{c}{$\begin{array}{c}\text { Gleason score } \geq 7 \text { at TZ } \\
\text { (lesions) }\end{array}$} & \multicolumn{3}{c}{ Gleason score $\geq 7$ at PZ } \\
PI-RADS & 3 & 4 & 5 & 3 & 4 & 5 \\
\hline 1 & 1 & 1 & 4 & 0 & 7 & 13 \\
2 & 1 & 1 & 4 & 0 & 9 & 13 \\
3 & 1 & 0 & 3 & 0 & 6 & 15 \\
4 & 0 & 5 & 3 & 0 & 2 & 10 \\
\hline
\end{tabular}


Intermediate to good agreement was found among four observers, ICC $=0.542,(95 \% \mathrm{CI}: 0.009,0.982)$ between moderate to high grade lesion (Gleason score $\geq 7$ ) in $\mathrm{PZ}$ only and given PI-RADS score of all observers Table 10.

\section{Discussion}

The findings of our study revealed that the new version of PI-RADS v2 provided intermediate to good inter-observer agreement among readers of varying experience as well as excellent agreement among four observers, ICC $=0.972$, (95\% CI: $0.915,0.995)$ between location of tumor ( $\mathrm{TZ}$ only, PZ only, both TZ and PZ) and low grade to high grade lesion (Gleason score $<7$ and $\geq 7$ ). In 2013, the Rosenkrantz et al. ${ }^{(18)}$ showed that the inter-observer reproducibility for the original PI-RADS was moderate (concordance correlation coefficient, 0.47) among readers of varying experience, similar to the results of our study. Our study showed the most significantly correlated PI-RADS assessment between observers 1 and 2 followed by observers 1 and 3 . Observer 1 was the most experienced followed by observers 2,3 and 4. A slight difference was found between the result (including location of tumor and given PI-RADS score) of observer 4 who was least experienced and the results of more experienced observers. Our results suggest that experience is necessary for further improving the detection of prostate cancer by MRI imaging and can lead to a fairly high level of accuracy. MR is gaining acceptance as the most accurate imaging investigation to locally assess prostate cancer. The improved performance of MR imaging within the last several years has probably been due mainly to advances in MR technology. Understanding imaging criteria and experience in image interpretation are also growing. In 2010, Akin et al. ${ }^{(20)}$ showed that dedicated interactive training sessions conducted by expert radiologists who provided weekly interactive tutorials with individualized feedback incorporating pathologic results of previously interpreted studies significantly improved accuracy in detecting tumors and extracapsular extensions among radiology fellows. In a similar fashion, Leeuwenburgh et al. ${ }^{(21)}$ showed the efficacy of a tutorial that provided direct feedback to radiologists after interpreting each case to improve performance in diagnosing acute appendicitis. To our knowledge, the lesion revealed Gleason score $\geq 7$ defined as moderate to high grade lesion. Our study showed excellent inter-observer agreement,
ICC $=0.903,(95 \% \mathrm{CI}: 0.575,0.997)$ and intermediate to good inter-observer agreement, ICC $=0.542$, (95\% CI: $0.009,0.982$ ) between moderate to high grade lesions in $\mathrm{TZ}$ only and in PZ only, respectively. In 2013, Schimmöller el al. ${ }^{(4)}$ evaluated the original PI-RADS from 2012 and showed higher accuracy in the PZ than in the TZ. In our study, the results for the two zones were comparable. This might have indicated better performance for detecting tumors in the $\mathrm{TZ}$ with the revised edition and generally increased awareness of anterior lesions in the TZ. However, the size of tumor is important, as larger tumors can be more easily detected in TZ. No significant relationship was found between incidence of location of tumor and low to high grade lesion (Gleason score $<7$ and $\geq 7$ ). This might be due to our small sample size.

Our study had limitations. First, because our study was retrospective, selection and verification biases may have occurred. Moreover, because readers interpreted the MR imaging data with the knowledge that the patients had prostate cancer, a potential bias might have been readers considering equivocal lesions as prostate cancer; thereby, increasing the sensitivity. Second, because our study included patients who underwent radical prostatectomy, a selection bias might have been that only patients with less aggressive and more localized prostate cancer were involved. Finally, the correlation between imaging and histologic examination on a section-by-section basis has inherent limitations because the angle of the histologic slices may differ from that at MR imaging and the prostate usually shrinks during fixation.

\section{Conclusion}

PI-RADS are an important standardization tool for reporting multiparametric MR imaging results. However, the results of this study were intermediate to good inter-observer agreement for readers of varying experience showing like the first version of PI-RADS. The inter-observer agreement may be increased by additional divisions of PI-RADS atlas and diminished variation in the acquisition, interpretation and reporting of prostate mp MRI examinations.

\section{References}

1. Ferlay J, Shin HR, Bray F, Forman D, Mathers C, Parkin DM. Estimates of worldwide burden of cancer in 2008: 
GLOBOCAN 2008. Int J Cancer 2010; 127: 2893-917.

2. Barentsz JO, Richenberg J, Clements R, Choyke P, Verma $S$, Villeirs $G$ et al. ESUR prostate MR guidelines 2012. Eur Radiol 2012; 22: 746-57.

3. American College of Radiology. Breast Imaging Reporting and Data System (BI-RADS), Breast Imaging Atlas, 4th edition American College of Radiology (ACR) 2003, Reston.

4. Schimmöller L, Quentin M, Arsov C, Lanzman RS, Hiester A, Rabenalt R et al. Inter-reader agreement of the ESUR score for prostate MRI using in-bore MRIguided biopsies as the reference standard. Eur Radiol 2013; 23: 3185-90.

5. Mistry K, Cable G. Meta-analysis of prostate-specific antigen and digital rectal examination as screening tests for prostate carcinoma. J Am Board Fam Pract 2003; 16: 95-101.

6. Heidenreich A, Aus G, Bolla M, Joniau S, Matveev $\mathrm{VB}$, Schmid HP et al. EAU guidelines on prostate cancer. Eur Urol 2008; 53: 68-80.

7. Dickinson L, Ahmed HU, Allen C, Barentsz JO, Carey $\mathrm{B}$, Futterer JJ et al. Magnetic resonance imaging for the detection, localization and characterisation of prostate cancer: recommendations from a European consensus meeting. Eur Urol 2011; 59: 477-94.

8. Umbehr M, Bachmann LM, Held U, Kessler TM, Sulser

$\mathrm{T}$, Weishaupt $\mathrm{D}$ et al. Combined magnetic resonance imaging and magnetic resonance spectroscopy imaging in the diagnosis of prostate cancer: a systematic review and meta- analysis. Eur Urol 2009; 55: 575-90.

9. 9. Mazaheri Y, Shukla-Dave A, Muellner A, Hricak H. MR imaging of the prostate in clinical practice. MAGMA 2008; 21: 379-92.

10. Hoeks CM, Barentsz JO, Hambrock T, Yakar D, Somford DM, Heijmink SW et al. Prostate cancer: multi-parametric MR imaging for detection, localization, and staging. Radiology 2011; 261: 46-66.

11. Portalez D, Rollin G, Leandri, Elman B, Mouly P, Jonca F et al. Prospective comparison of T2w-MRI and dynamic-contrast-enhanced MRI, 3D-MR spectroscopic imaging or diffusion-weighted MRI in repeat TRUSguided biopsies. Eur Radiol 2010; 20: 2781-90.

12. Hricak H, Choyke PL, Eberhardt SC, Leibel SA, Scardino PT. Imaging prostate cancer: a multidisciplinaryperspective. Radiology 2007; 243: 28-53.
13. Röthke M, Blondin D, Schlemmer HP, Franeil T, PI-RADS classification: structured reporting for MRI of the prostate. Rofo 2013; 185: 253-61.

14. Franiel T, Stephan C, Erbersdobler A, Dietz E, Maxeiner A, Hell $\mathrm{N}$ et al. Areas suspicious for prostate cancer: MR-guided biopsy in patients with at least one transrectal US-guided biopsy with a negative finding multiparametric MR imaging for detection and biopsy planning. Radiology 2011; 259: 162-72.

15. Kitajima K, Kaji Y, Fukabori Y, Yoshida K, Suganuma $\mathrm{N}$, Sugimura K. Prostate cancer detection with $3 \mathrm{~T}$ MRI: comparison of diffusion-weighted imaging and dynamic contrast-enhanced MRI in combination with T2- weighted imaging. J Magn Reson Imaging 2010; 31: 625-31.

16. Fütterer JJ, Heijmink SW, Scheenen TW, Veltman J, Huisman HJ, Vos P et al. Prostate cancer localization with dynamic contrast- enhanced MR imaging and proton MR spectroscopic imaging. Radiology 2006; 241: 449-58.

17. Tanimoto A, Nakashima J, Kohno H, Shinmoto H, Kuribayashi S. Prostate cancer screening: the clinical value of diffusion- weighted imaging and dynamic MR imaging in combination with T2-weighted imaging. J Magn Reson Imaging 2007; 25: 146-52.

18. Rosenkrantz AB, Lim RP, Haghighi M, Somberg MB, Babb JS, Taneja SS. Comparison of interreader reproducibility of the prostate imaging reporting and data system and likert scales for evaluation of multiparametric prostate MRI. Am J Roentgenol 2013; 201: W612-8.

19. ACR, ESUR, AdMeTech Foundation. Prostate Imaging and Reporting and Data System: Version 2. 2014. Available at:14/4/2015, http://www.acr.org/w/media/ACR/Documents/PDF/QualitySafety/Resources/PIRADS/PIRADS \%20V2.pdf[accessed 14.04.15].

20. Akin O, Riedl CC, Ishill NM, Moskowitz CS, Zhang J, Hricak H. Interactive dedicated training curriculum improves accuracy in the interpretation of MR imaging of prostate cancer. Eur Radiol 2010; 20: 995-1002.

21. Leeuwenburgh MM, Wiarda BM, Bipat S, Nio CY, Bollen TL, Kardux JJ et al. Acute appendicitis on abdominal MR images: training readers to improve diagnostic accuracy. Radiology 2012; 264: 455-63. 